\title{
A new parameter tuning approach for enhanced motor imagery EEG signal classification
}

Shiu Kumar ${ }^{1,2} \cdot$ Alok Sharma ${ }^{2,3,4}$

\begin{abstract}
A brain-computer interface (BCI) system allows direct communication between the brain and the external world. Common spatial pattern (CSP) has been used effectively for feature extraction of data used in BCI systems. However, many studies show that the performance of a BCI system using CSP largely depends on the filter parameters. The filter parameters that yield most discriminating information vary from subject to subject and manually tuning of the filter parameters is a difficult and time consuming exercise. In this paper, we propose a new automated filter tuning approach for motor imagery electroencephalography (EEG) signal classification, which automatically and flexibly finds the filter parameters for optimal performance. We have evaluated the performance of our proposed method on two public benchmark datasets. Compared to the existing conventional CSP approach, our method reduces the average classification error rate by $2.89 \%$ and $3.61 \%$ for BCI Competition III dataset IVa and BCI Competition IV dataset I, respectively. Moreover, our proposed approach also achieved lowest average classification error rate compared to state-of-the-art methods studied in this paper. Thus, our proposed method can be potentially used for developing improved BCI systems, which can assist people with disabilities to recover their environmental control. It can also be used for enhanced disease recognition such as epileptic seizure detection using EEG signals.
\end{abstract}

Keywords Brain-computer interface $(\mathrm{BCI}) \cdot$ Filter tuning $\bullet$ Genetic algorithm (GA) • Motor imagery (MI) • Temporal filters

Alok Sharma*

alok.sharma@griffith.edu.au

1 Department of Electronics, Instrumentation \& Control, School of Electrical \& Electronics Engineering, Fiji National University, Fiji.

2 School of Engineering and Physics, Faculty of Science, Technology \& Environment, The University of the South Pacific, Fiji.

3 Institute for Integrated and Intelligent Systems (IIIS), Griffith University, Brisbane, Australia.

4 Laboratory for Medical Science Mathematics, RIKEN Center for Integrative Medical Sciences, Yokohama 230-0045, Japan

\section{Introduction}

A brain computer interface (BCI) system can bridge the gap between humans and computers by translating thoughts into control signals, which can be used to control external devices [24]. It can be used to assist severely disabled people to live a quality life with minimum or no dependence. Motor imagery (MI) is an important paradigm in developing a BCI system that allows direct human-computer interaction (HCI) i.e. allowing communication between the brain and the external devices without the involvement of peripheral nerves and muscles [24]. BCI research is increasingly gaining interest as it aims to re-establish independence and reduce social exclusion for people with disabilities. BCI systems have been explored for a wide range of applications such as communication control [12], movement control [20], environment control [5], neurorehabilitation $[9,22,30,31,43]$, P300-based word typing system [1], biometric identification [18], and brain wave controlled robots and wheelchairs for the disabled people [23, 28]. Biomedical engineering is the major focus and emphasis of current BCI research [9, 14-16, 21, 22, 30, 42, 44, 51, 53, 58].

Research in the field of MI-BCI systems is ongoing as there are still many problems that exist and are yet to be addressed properly. Focus is also on developing more accurate, reliable and computationally efficient MI-BCI systems that can be incorporated with wearable devices. A range of approaches have been reported for solving different aspects of the problem.

CSP has been widely used in MI-BCI applications as it aims to find the spatial filters that maximize the variance of one class, while minimizing the variance of the other class in order to discriminate between different classes. CSP was first introduced for a two class problem and later extended to multi-class problem. The conventional CSP approach uses a single wide frequency band mostly in the range of $8-30 \mathrm{~Hz}$, covering both the mu and beta rhythms. However, certain frequency components are useless resulting in degrading the overall performance of the system. To improve the performance of the conventional CSP approach, common spatio-spectral pattern (CSSP) [19] and common sparse spectral spatial pattern (CSSSP) [7] methods were proposed. 
The responsive frequency bands vary from subject to subject and to address this problem, the use of multiple frequency bands has been proposed [3, 25, 46]. In [25], a sub-band CSP (SBCSP) method has been proposed. This paper uses multiple filters (filter banks) to decompose the signal into multiple bands called sub-bands. CSP is then performed separately on each of these sub-bands to extract the variance based CSP features. Linear discriminant analysis (LDA) is then performed on each feature set obtained from the separate sub-bands in order to reduce the feature dimensionality of each sub-band to one. Thus one dimensional features of each sub-band (LDA scores) are fused together and fed into a support vector machine (SVM) classifier. The method performed well and outperformed the CSP, CSSP and CSSSP methods. The shortfall of the SBCSP method is that since a number of sub-bands are used, the number of extracted features also increased some of which are not useful and tend to degrade the performance of the system. To address this issue, filter bank CSP (FBCSP) [3] has been proposed, in which all the variance based CSP features from each sub-band is combined together and different feature selection and classification methods have been evaluated. Feature selection is employed in order to select the significant features thereby removing the redundant features. Although FBCSP outperformed the SBCSP method, it still utilized several sub-bands that lead to higher computational cost. To address this issue, a discriminative filter bank CSP (DFBCSP) [46] method has been proposed. In DFBCSP, the signals are decomposed into multiple sub-bands (12 sub-bands in the frequency range of $6-40 \mathrm{~Hz}$ ) during training phase. Fisher's ratio of the bands spectral power of channel C3 is then used to select the top 4 sub-bands that give maximum discrimination between the different tasks. Channel C3 located on the contralateral hemisphere was selected as signals from sensorimotor cortex have shown to have maximum discriminating power amongst several MI tasks. Signals from contralateral channels C3, C4, Cz and its surrounding channels were used for estimating the Fisher's ratio. The authors discovered that single channel $\mathrm{C} 3$ or $\mathrm{C} 4$ alone offered superior performance in selecting the sub-bands and thus channel $\mathrm{C} 3$ has been used. The selected sub-bands are then used for extracting the variance based CSP features and fed into an SVM classifier for classification. The method achieved a reduced computational cost compared to SBCSP and FBCSP and also performed better.

To address the issue of computation cost due to the use of large number of sub-bands and due to the fact that some sub-bands may contain redundant information, a sub-band binary particle swarm optimization approach
(BPSO) using CSP [49] has been proposed. In sub-band BPSO-CSP, the EEG signals are decomposed into subbands using multiple filter banks as in DFBCSP. Then the BPSO algorithm is employed to select the sub-bands that give optimal performance using the training data. Recently, a sparse filter band CSP (SFBCSP) [56] method that uses multiple filter bands is proposed, which optimizes the sparse patterns. Bayesian learning has also gained increased attention recently and has been used for feature selection in various applications [45, 57]. In [54], a sparse Bayesian learning approach (SBLFB) has been used for obtaining sparse features that are used for MI EEG signal classification. A number of studies have explored feature optimization and classifier optimization for improved EEG signal classification in BCI applications. A method based on multi-kernel extreme learning machine (MKELM) [55] has been proposed for EEG signal classification. Gaussian and polynomial kernels have been integrated together with multi-kernel learning approach for more robust classification of multiple non-linear feature spaces. In [48], a spatiotemporal feature extraction in conjunction with multivariate linear regression has been proposed, which improved the classification performance by learning discriminative steady-state visual evoked potential (SSVEP) features. A number of component analysis methods have also been proposed for processing biomedical data such as independent component analysis (ICA), blind source separation and matrix-based component analysis methods [58].

During MI, the power of the EEG signal in certain specific regions of the brain decreases and later increases as the MI concludes. These physiological phenomena are known as event related desynchronization (ERD) and event related synchronization (ERS), respectively [26]. ERD and ERS are directly related to sensory motor rhythms mu and beta. The mu $(8-12 \mathrm{~Hz})$ and beta $(18-25$ $\mathrm{Hz}$ ) rhythms are excellent features for MI signals. However, the frequency band varies across different subjects together with the varying psychological states of the subjects [2]. CSP has been widely explored and used effectively for formulating spatial filters in ERD/ERS detection [29]. Although CSP has performed considerably well for spatial feature extraction, the filtering of signals in the time domain confines its performance. Using improper frequency range for the filter or simply using unfiltered EEG signal generally yields undesirable classification results [25]. Thus, while employing CSP, usually a broad frequency band is used or the filters are manually tuned for each of the subjects [17]. These approaches are not suitable for obtaining optimal performance as the wide band contains certain 
frequency ranges that are not useful. On the other hand, manually tuning is a time consuming and difficult approach. When filtering is performed on raw data, the filter parameters need to be selected carefully [50].

To address the problem of selecting subject-specific frequency band that achieves optimal performance using the CSP algorithm, this paper proposes the temporal filter parameter optimization with CSP approach (TFPOCSP predictor). A BCI system usually involves four phases: 1) temporal filtering of the EEG data, 2) feature extraction, 3) feature selection and 4) classification. In this work, we mainly focused on phase 1 i.e. temporal filtering to obtain the optimum parameters and other 3 phases will be implemented as in CSP approach. We also aimed to employ a single bandpass filter in a quest to keep the computation complexity of the resulting system to a minimum. Generally in phase 1 the signal is filtered either using single or multiple bandpass filters. Spatial filtering is performed and features are extracted in phase 2. The third phase is usually incorporated to select the most discriminating features in order to improve the system performance. The final phase involves using a suitable classifier to classify the EEG signals. The public benchmark dataset IVa of BCI competition III is used to show that the proposed approach can be used to select the appropriate subject-specific frequency band for optimal performance. The effectiveness of the proposed TFPO-CSP predictor over the state-of-the-art methods such as FBCSP, DFBCSP and SBLFB is demonstrated.

The following sections of the paper are organized as follows. Section 2 presents the proposed TFPO-CSP predictor while in Section 3 and 4 the experimental study and findings are presented and discussed. Section 5 draws the conclusion and gives insight of some future works.

\section{Methods}

\subsection{Description of the Datasets}

The public benchmark Dataset IVa of BCI Competition III [6] and Dataset I of BCI Competition IV [4] (referred to as dataset 1 and dataset 2 from here onwards, respectively) have been used for evaluating the effectiveness of the proposed approach.

Dataset 1 contains 118 channels of EEG signals for right hand and left foot MI tasks, which have been recorded from five healthy subjects labeled $a a, a l, a v$, $a w$, and ay. The down sampled signal at $100 \mathrm{~Hz}$ has been used. It contains 140 trials of each task for each of the subjects. A detail description of the dataset can be found online at [http://www.bbci.de/competition/iii/].

Dataset 2 contains 59 channels of real long-term EEG signals recorded for left hand, and right hand MI tasks acquired from 7 healthy subjects (named $a$ to $g$ ). The down sampled signal at $100 \mathrm{~Hz}$ has been used, which contains 200 trials for each subject with equal number of each type of MI tasks. A detail description of the dataset can be found online at [http://www.bbci.de/competition/iv/].

\subsection{Experimental Setup}

In this study, we have extracted the EEG data between 0.5 seconds and 2.5 seconds (i.e. 200 time points) after the visual cue. The number of CSP filters is set to 6 (i.e., $m=3$ ) in all experiments, except for the DFBCSP and SBLFB methods in which two CSP filters have been used (i.e., $m=1$ ). In all experiments (except for the proposed approach), an $8^{\text {th }}$ order butterworth bandpass filter has been used and SVM is used as the classifier. The following experimental settings have been used for each of the methods:

- CSP: A butterworth bandpass filter with passband of 7-30 Hz has been applied.

- CSSP: Bandpass filter is the same as in CSP. The sample point delay $\tau$ value was chosen from 1 to 15 (as in [8]), which was selected by performing 10-fold cross validation on the train data. The average error rate of the 10 -fold cross validation for each value of $\tau$ is calculated and the value of $\tau$ giving the minimum error rate is selected for testing.

-FBCSP: Six bandpass filters having bandwidth of 6 $\mathrm{Hz}$ in the range of $4 \mathrm{~Hz}$ to $40 \mathrm{~Hz}$ with no overlap have been used. These were adopted from Higashi and Tanaka [8], as using these settings gave optimal results. Mutual information based feature selection has been performed as it gave the best results in [3].

-DFBCSP: Twelve bandpass filters with bandwidth of $4 \mathrm{~Hz}$ in the range of 6 to $40 \mathrm{~Hz}$ have been used, as described in [46]. The top four bands were selected using Fisher's ratio and further utilized for classification.

-SFBCSP: Seventeen bandpass filters having bandwidth of $4 \mathrm{~Hz}$ with an overlap of $2 \mathrm{~Hz}$ in the range of $4-40 \mathrm{~Hz}$ have been used, as in [56]. The regularization parameter $\lambda$ was chosen using 10 -fold cross validation.

- $S B L F B$ : As in [54], seventeen bandpass filters having bandwidth of $4 \mathrm{~Hz}$ with an overlap of $2 \mathrm{~Hz}$ in the range of $4-40 \mathrm{~Hz}$ have been used. Multiple sub-band features are learned by SBLFB. 
- BPSO-CSP: Ten bandpass filters having bandwidth of $4 \mathrm{~Hz}$ in the range of $8 \mathrm{~Hz}$ to $30 \mathrm{~Hz}$ with an overlap of $2 \mathrm{~Hz}$ have been used. Only 25 selected channels of data were used for processing as in Wei and Wei [49].

- TFPO-CSP (PSO): Uses particle swarm optimization (PSO) as the optimization algorithm. The number of particles for the PSO algorithm is set to 10 because with 10 particles the optimal solution is found in a reasonable amount of time. The dimension of each particle is set to 3 , where dimensions represent the lower cutoff frequency, upper cutoff frequency and filter order of the butterworth bandpass filter. The range of the dimensions were set as follows: lower cutoff frequency $[0.50 \mathrm{~Hz}, 16.0 \mathrm{~Hz}]$, upper cutoff frequency $[18 \mathrm{~Hz}, 32 \mathrm{~Hz}]$, and filter order $[1,30]$. The frequency ranges were selected so as to include the frequencies of the mu and beta rhythms. The range for the velocity was set to $[-4,4]$. The maximum number of iterations $\left(I_{\max }\right)$ was set to 35 , which was decided after carrying out a number of experiments. In TFPO-CSP (PSO), different subjects have different fitness condition values. Thus, several trial runs were conducted without the use of fitness condition in order to determine the minimum error rate that can be achieved by the TFPO-CSP (PSO) for each subject. It was noted that the minimum error rates that could be obtained for each subject was similar to the best error rate that can be achieved amongst the DFBCSP and SBLFB methods. Therefore, the fitness condition value used for each subject was set to the minimum of the error rates obtained amongst the DFBCSP and SBLFB methods.

- TFPO-CSP (GA): Uses genetic algorithm (GA) as the optimization algorithm. The frequency ranges, filter order, maximum number of iterations and fitness conditions were same as that used for TFPO-CSP (PSO). The population size was set to 10 , which is same as the number of particles used for TFPO-CSP (PSO). Tournament selection has been employed with tournament size of 3 . A crossover percentage of 0.7 and mutation percentage of 0.3 have been used. Fitness based survivor selection is performed. For BCI competition III dataset IVa, the fitness conditions were set to $10,1.5,25,5.0$ and 4.5 for subjects $a a, a l$, $a v, a w$ and $a y$, respectively, while for BCI competition IV dataset I the fitness conditions were set to $13,41.5,33,21,11,14$, and 8 for subjects $a$ to $g$, respectively.

-TFPO-CSP (GA)*: All experimental settings were same as TFPO-CSP (GA), except that only 25 selected channels of data as used in BPSO-CSP were used for processing in order to compare our proposed method with BPSO-CSP.

-TFPO-CSP (ABC): Uses artificial bee colony (ABC) as the optimization algorithm. The frequency ranges, filter order, maximum number of iterations and fitness conditions were same as that used for TFPOCSP (PSO). The colony size was set to 10 , which is same as the number of particles used for TFPO-CSP (PSO).

\subsection{Proposed TFPO-CSP method}

The framework for the proposed TFPO-CSP predictor is presented in Fig. 1. The temporal filtering of the MI EEG signal is integral because CSP mostly depends on responsive frequency band in order to perform well. Unfiltered signal or filtering the signal with inappropriate frequency band would result in poor performance of the system. Moreover, the responsive frequency band varies from subject to subject and thus using a fixed frequency band is detrimental in achieving a system with optimal performance. Therefore, in order to find the optimum subject-specific responsive frequency band, GA has been employed for finding optimal filter band parameters. The proposed TFPO-CSP predictor involves 4 phases: temporal filter parameter selection and filtering, spatial filtering, feature extraction, and classification.

Fig. 1 Framework for the proposed TFPO-CSP predictor with $\mathrm{GA}$ as the optimization algorithm

The first phase that involves selecting the temporal filter parameters using $\mathrm{GA}$ is the major focus of this paper as it aims to find a single responsive filter, which can give optimal results. The GA has been modified and used with our proposed system in order to select the best parameters (upper cutoff frequency, lower cutoff frequency, and filter order) of a bandpass butterworth filter. Once the filter parameters are selected using GA, the raw EEG signal is bandpass filtered using the selected filtering parameters. In the second phase, CSP is utilized for spatial filtering. The variance based CSP features are then extracted from the spatially filtered data during the third phase, while classification using SVM classifier is performed in the final phase. The following sub-sections present each of the phases in more detail.

The ABC [10] and PSO [27] algorithms have also been modified to be used as the optimization algorithm in our proposed predictor for finding the optimal parameters. Details of the ABC and PSO algorithms are given in supplementary materials. 


\subsubsection{Optimization of temporal filter parameters}

For optimizing the parameters of the temporal filter, we have employed GA [52]. GA is based on natural selection and genetics, and is mostly used for optimization and search problems. A population of chromosomes (solutions) is generated with dimension equal to the number of parameters to be optimized. This can be done either randomly or by heuristic initialization. The first generation is referred to as the parents. Children are then generated from selected parents using crossover. The selection of parents can be done using various methods such as Roulette wheel selection, stochastic universal sampling (SUS), tournament selection, rank selection and random selection. Mutation is then performed on a number of randomly selected parents to form new mutants. Finally, survivor selection is done and the chromosomes that survive become the parents for the next iteration. This process is repeated until the desired fitness condition or the maximum number of iterations is reached. The pseudo code of the proposed framework is given in Algorithm 1 and 2. The fitness function value referred to in Algorithm 2 is the error returned by Algorithm 1 .

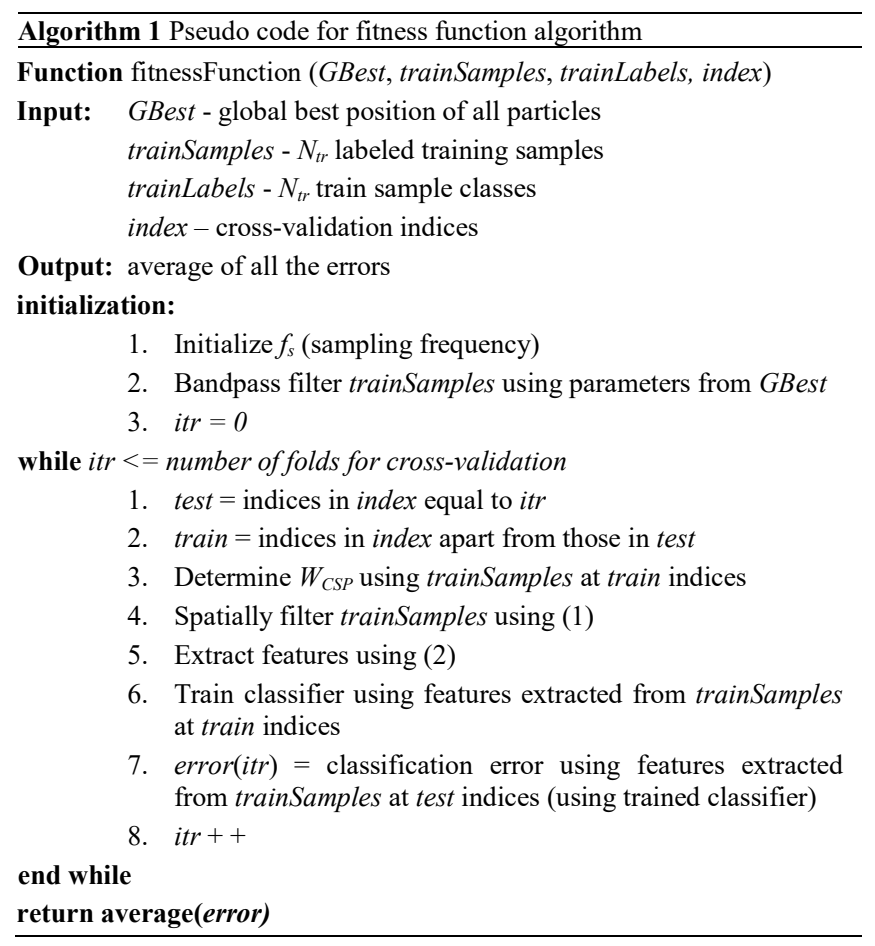

\subsection{CSP Feature Extraction}

The CSP technique has gained a lot of attention and has been widely used in recent years for MI-BCI systems since its inception, where it has been used for detection of abnormalities in EEG signal [13]. CSP projects the data to a new time series maximizing the variance of one class, while minimizing the variance of the other class. A detailed explanation of the CSP algorithm can be found in our previous work [17]. Consider the bandpass filtered EEG sample $X_{n} \in R^{C \times T}$, where $n$ denotes the $n$-th sample, $\mathrm{C}$ is the number of channels and $\mathrm{T}$ is the number of time points. The spatially filtered signal $Z_{n}$ can be obtained using (1), where $W_{c s p}$ is the CSP spatial filter that is formed by selecting the first and last $m$ columns of CSP matrix, $W$.

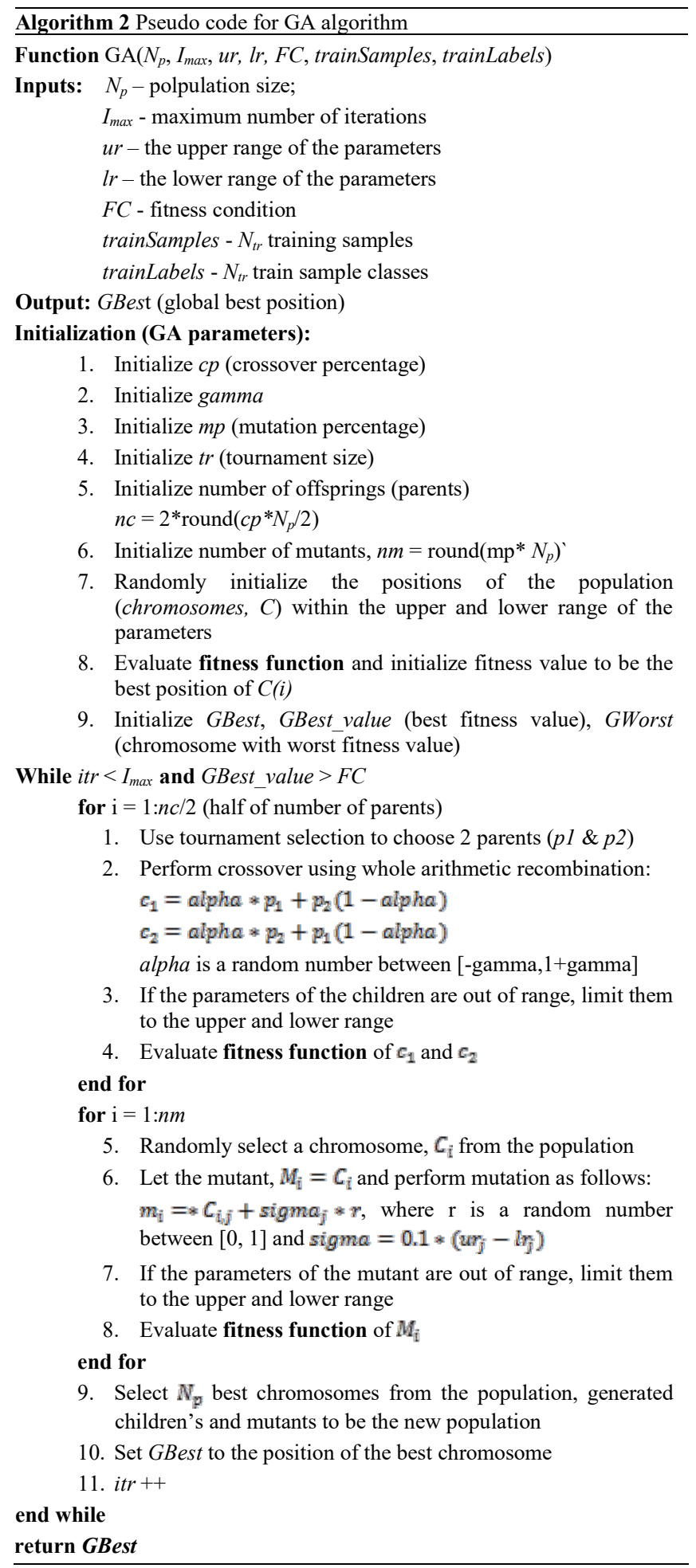




$$
Z_{n}=W_{C S P}^{T} X_{n}
$$

The variance based CSP features of $n$-th sample is then extracted using (2), where $F_{n}^{i}$ is the $i$-th feature of the $n$ th sample, and $\operatorname{var}\left(Z_{n}^{j}\right)$ denotes the variance of $j$-th row of $Z_{n}$.

$$
F_{n}^{i}=\log \left(\frac{\operatorname{var}\left(Z_{n}^{i}\right)}{\sum_{j=1}^{2 m} \operatorname{var}\left(Z_{n}^{j}\right)}\right)
$$

Using the extracted features of each sample, the feature set is constructed using (3), where $N$ is the number of samples. Thus the features of training and test samples are used to construct the training and test feature sets, respectively.

$$
F=\left[\begin{array}{cccc}
F_{1}^{1} & F_{1}^{2} & \cdots & F_{1}^{2 m} \\
\vdots & \vdots & \ddots & \vdots \\
F_{N}^{1} & F_{N}^{2} & \cdots & F_{N}^{2 m}
\end{array}\right]
$$

\subsection{Classification}

An SVM classifier with linear kernel is trained using the training feature set. The test feature set is then used to classify each of the test samples using the trained SVM classifier model.

\section{Results}

\subsection{Evaluation scheme}

The 10x10-fold cross-validation scheme is used to evaluate the performance of all experiments conducted. The trials are divided into 10 sets. One set is selected as test set while all others are selected as training sets. Only the training set is used by GA algorithm to find the best temporal filter parameters. The fitness function again divides the training set of GA algorithm into 10 sets for performing 10-fold cross-validation. The average of these cross-validation values are returned by the fitness function, i.e. the average classification error rate. Thus, the GBest chromosome returned by GA is used to test the performance of the initial test set. This procedure is repeated 10 times with a different set used as test data and remaining used as training data of GA. The whole procedure was repeated 10 times and the average of these classification error rates are presented as results.

\subsection{Experimental Result Analysis}

The proposed TFPO-CSP predictor searches for the optimal frequency band and filter order. It can be observed (and suggested in the literature) that the responsive frequency bands vary between subjects, and thus different band leads to different outcome. It is therefore important to give due consideration to band optimization. Thus, in order to improve the performance, one aspect is to evaluate bands using the training dataset. We have evaluated the effect of filter order on the performance of the BCI system in Fig. 2. It shows the 10 -fold cross-validation error rate for all the subjects of dataset 1. It can be observed from Fig. 2 that the filter order has a considerable effect on the overall error rate. Similar results were obtained for dataset 2 (refer to Figure S1 of supplementary material). This explains the reason for optimizing the filter order together with the frequency band. Since the classification error rates are high for larger values of filter order, we have limited the filter order in the range between 1 and 30 .

Fig. 2 Average error rates of 10 -fold cross validation with different values of filter order (results obtained using dataset 1)

Two separate experiments were carried out to evaluate the performance of the proposed system. In the first experiment, we selected a subset of 25 channels (as in [49]) that are instrumental for the neurophysiological discrimination between the tasks. In the second experiment, all channels of data were used. The results obtained using dataset 1 and dataset 2 are shown in Fig. 3 and Fig. 4, respectively. The TFPO-CSP (GA)* indicates the results obtained when selected subset of 25 channels are used, while the TFPO-CSP (GA) indicates the result when all channels data are used for processing.

Fig. 3 10x10-fold cross-validation error rates for different methods using public benchmark dataset 1

Fig. 4 10x10-fold cross-validation error rates for different methods using public benchmark dataset 2

The results obtained shows that the proposed TFPOCSP predictor outperformed state-of-the-art methods studied in this paper in terms of the average classification error rates using either of the three optimization algorithms (PSO, GA, or ABC). The TFPO-CSP (ABC) and TFPO-CSP (PSO) methods obtained average error rates that were within $\pm 1 \%$ of the average error rate obtained using TFPO-CSP (GA). Furthermore, our proposed method also performed better than the MKELM approach that achieved average classification error rate of $12.50 \%$, evaluated on dataset 1. A spatial-frequency-temporal optimized feature sparse representation-based classification (SFTOFSRC) method is proposed in [21] which achieved error rate of $23.89 \%$ on dataset 1. The SFTOFSRC method used multiple frequency bands and multiple time segments. It also employed channel selection. While the authors showed 
that their method improves performance when sparse regression classification (SRC) with dictionary optimization is used, it obtained a considerable higher classification error rate. This could be due to the preprocessing that may have been used. On the other hand, the TFPO-CSP method achieved average classification error rate of $10.19 \%$ with common averaging as the only pre-processing step.

Using only selected 25 channels, we were able to achieve promising results and obtained the minimum average classification error rate in comparison with CSP, CSSP, FBCSP and BPSO-CSP. It can be noted that our proposed predictor with 25 channels (TFPO-CSP (GA)*) also shows very promising results. Therefore, the proposed approach is also recommended over other competing methods when computation complexity is a requirement, where data from fewer channels could be used. For dataset 2, subjects $a$ and $c$ achieved the minimum error rate using TFPO-CSP (GA) while subjects $e$ and $f$ achieved the minimum error rates using TFPO-CSP (ABC). The minimum average error rate was obtained using TFPO-CSP (PSO) and TFPO-CSP (ABC) for dataset 1 and dataset 2, respectively. However, there was no significant difference compared to the average error rate of TFPO-CSP (GA) (less than $0.5 \%$ difference in error rate). The SBLFB method also performed well on dataset 2 with subjects $b, d$ and $g$ achieving the minimum error rate. The average error rate is also comparable to that of the proposed method, however, it did not perform well on dataset 1 .

Tables 1 and 2 shows the ranges in which the lower and upper cutoff frequencies and filter orders of the butterworth bandpass filter that were selected using the TFPO-CSP (PSO), TFPO-CSP (GA) and TFPO-CSP $(\mathrm{ABC})$ approaches for dataset 1 and dataset 2, respectively. For dataset 1 and dataset 2, it can be noted that the lower cutoff frequency range for all the subjects is in the range of 4-15 Hz, the upper cutoff frequency is in the range of $19-32 \mathrm{~Hz}$ and the filter order is in the range of 1-30. The range of values (for lower and upper cutoff frequency and filter order) that were obtained using the TFPO-CSP (PSO), TFPO-CSP (ABC) and TFPO-CSP (GA) were similar. All the frequency ranges are close to the frequency range of $8-30 \mathrm{~Hz}$ that includes both the mu and beta rhythms. It is clearly shown that the responsive frequency bands vary from subject to subject as the range of frequency bands selected for different subjects vary.

The average, minimum and maximum number of iterations required by the TFPO-CSP (PSO), TFPO-CSP $(\mathrm{ABC})$ and TFPO-CSP (GA) approaches to select the temporal filter parameters for each subject are shown in
Tables 3 and 4 for dataset 1 and dataset 2, respectively. The minimum number of iterations required by the TFPO-CSP (GA) approach is 1 for all the subjects. The average number of iterations required for selecting the temporal filter parameters using the TFPO-CSP (GA) approach was quite low for all subjects $(\leq 3)$ except for subject $a a$ of dataset 1 (having value of 10). For subjects $a a$ and $a w$ of dataset 1 (using TFPO-CSP (PSO)) and subject $a$ of dataset 2 (using TFPO-CSP (ABC)), the maximum number of iterations is the maximum number of iteration set during the experiment (i.e. 35). This may be due to the desired fitness condition set for subject $a a$ and $a w$ of dataset 1 and subject $a$ of dataset 2 being challenging or due to a local minimum that resulted in the TFPO-CSP (PSO) and TFPO-CSP (ABC), respectively, taking longer time to find the desired solution. TFPO-CSP (GA) obtained the least number of average iterations required to search for the optimal parameters.

Table 1 Ranges of parameters that were selected by TFPOCSP (PSO), TFPO-CSP (GA) and TFPO-CSP (ABC) approach for the different subjects (Dataset 1)

\begin{tabular}{|c|c|c|c|c|c|c|}
\hline \multirow{2}{*}{ Parameter } & \multirow{2}{*}{ Method } & \multicolumn{5}{|c|}{ Subject } \\
\hline & & $a a$ & al & $a v$ & $a w$ & ay \\
\hline \multirow{3}{*}{$\begin{array}{l}\text { Lower cutoff } \\
\text { frequency }(\mathrm{Hz})\end{array}$} & $\begin{array}{l}\text { TFPO-CSP } \\
\text { (PSO) }\end{array}$ & $11-12$ & $4-11$ & $6-9$ & 8-11 & $4-10$ \\
\hline & $\begin{array}{l}\text { TFPO-CSP } \\
(\mathrm{ABC})\end{array}$ & $10-13$ & $3-12$ & $6-15$ & $8-12$ & $4-11$ \\
\hline & $\begin{array}{l}\text { TFPO-CSP } \\
\text { (GA) }\end{array}$ & $10-12$ & $6-12$ & $6-15$ & 8-11 & $5-10$ \\
\hline \multirow{3}{*}{$\begin{array}{l}\text { Upper cutoff } \\
\text { frequency }(\mathrm{Hz})\end{array}$} & $\begin{array}{l}\text { TFPO-CSP } \\
\text { (PSO) }\end{array}$ & $26-31$ & $20-28$ & $23-30$ & $24-32$ & $22-32$ \\
\hline & $\begin{array}{l}\text { TFPO-CSP } \\
(\mathrm{ABC})\end{array}$ & $23-32$ & $19-32$ & $24-32$ & $21-31$ & $20-32$ \\
\hline & $\begin{array}{l}\text { TFPO-CSP } \\
\text { (GA) }\end{array}$ & $27-32$ & $20-32$ & $25-31$ & $19-31$ & $22-32$ \\
\hline \multirow{3}{*}{ Filter order } & $\begin{array}{l}\text { TFPO-CSP } \\
\text { (PSO) }\end{array}$ & $17-30$ & $2-15$ & $2-29$ & $4-25$ & $4-10$ \\
\hline & $\begin{array}{l}\text { TFPO-CSP } \\
(\mathrm{ABC})\end{array}$ & $1-21$ & $1-30$ & $1-26$ & $3-22$ & $1-28$ \\
\hline & $\begin{array}{l}\text { TFPO-CSP } \\
\text { (GA) }\end{array}$ & $9-28$ & $1-29$ & $1-26$ & $2-21$ & $1-20$ \\
\hline
\end{tabular}

Table 2 Ranges of parameters that were selected by TFPOCSP (PSO), TFPO-CSP (GA) and TFPO-CSP (ABC) approach for the different subjects (Dataset 2)

\begin{tabular}{|c|c|c|c|c|c|c|c|c|}
\hline \multirow{2}{*}{ Parameter } & \multirow{2}{*}{ Method } & \multicolumn{5}{|c|}{ Subject } & \multirow[b]{2}{*}{$f$} & \multirow[b]{2}{*}{$g$} \\
\hline & & $a$ & $b$ & $c$ & $d$ & $e$ & & \\
\hline \multirow{3}{*}{$\begin{array}{l}\text { Lower } \\
\text { cutoff } \\
\text { frequency } \\
(\mathrm{Hz})\end{array}$} & $\begin{array}{l}\text { TFPO-CSP } \\
\text { (PSO) }\end{array}$ & $6-12$ & $7-14$ & $6-11$ & $6-12$ & $9-12$ & $7-10$ & $5-14$ \\
\hline & $\begin{array}{l}\text { TFPO-CSP } \\
(\mathrm{ABC})\end{array}$ & $8-12$ & $6-14$ & $5-11$ & $6-14$ & $8-11$ & $6-10$ & $2-15$ \\
\hline & $\begin{array}{l}\text { TFPO-CSP } \\
\text { (GA) }\end{array}$ & $7-11$ & $7-13$ & $6-10$ & $8-13$ & $9-12$ & $6-10$ & $4-15$ \\
\hline \multirow{3}{*}{$\begin{array}{l}\text { Upper } \\
\text { cutoff } \\
\text { frequency } \\
(\mathrm{Hz})\end{array}$} & $\begin{array}{l}\text { TFPO-CSP } \\
\text { (PSO) }\end{array}$ & $21-32$ & $22-31$ & $20-31$ & $24-30$ & $24-32$ & $22-30$ & $23-31$ \\
\hline & $\begin{array}{l}\text { TFPO-CSP } \\
(\mathrm{ABC})\end{array}$ & $20-31$ & $22-32$ & $20-32$ & $20-30$ & $22-32$ & $22-31$ & $23-32$ \\
\hline & $\begin{array}{l}\text { TFPO-CSP } \\
\text { (GA) }\end{array}$ & $20-32$ & $20-32$ & $21-32$ & $23-32$ & $22-32$ & $22-31$ & $21-32$ \\
\hline
\end{tabular}




\begin{tabular}{|c|c|c|c|c|c|c|c|c|}
\hline \multirow{3}{*}{ Filter order } & $\begin{array}{l}\text { TFPO-CSP } \\
\text { (PSO) }\end{array}$ & $2-25$ & $4-28$ & $4-22$ & $1-16$ & $3-19$ & $6-24$ & $1-21$ \\
\hline & $\begin{array}{l}\text { TFPO-CSP } \\
(\mathrm{ABC})\end{array}$ & -29 & $2-27$ & $1-24$ & $1-21$ & $1-14$ & $2-29$ & $1-24$ \\
\hline & $\begin{array}{l}\text { TFPO-CSP } \\
\text { (GA) }\end{array}$ & -24 & $1-30$ & $2-26$ & $1-22$ & $1-1$ & $2-23$ & $2-25$ \\
\hline
\end{tabular}

Table 3 The average, minimum and maximum number of iterations taken by TFPO-CSP (PSO), TFPO-CSP (GA) and TFPO-CSP (ABC) approach to select the temporal filter parameters (Dataset 1)

\begin{tabular}{|c|c|c|c|c|c|c|}
\hline \multirow{2}{*}{ Iterations } & \multirow{2}{*}{ Method } & \multicolumn{5}{|c|}{ Subject } \\
\hline & & $a a$ & $a l$ & $a v$ & $a w$ & ay \\
\hline \multirow{3}{*}{ Minimum } & $\begin{array}{l}\text { TFPO-CSP } \\
\text { (PSO) }\end{array}$ & 1 & 1 & 1 & 2 & 1 \\
\hline & $\begin{array}{c}\text { TFPO-CSP } \\
(\mathrm{ABC})\end{array}$ & 3 & 1 & 1 & 1 & 1 \\
\hline & $\begin{array}{l}\text { TFPO-CSP } \\
\text { (GA) }\end{array}$ & 1 & 1 & 1 & 1 & 1 \\
\hline \multirow{3}{*}{ Average } & $\begin{array}{l}\text { TFPO-CSP } \\
\text { (PSO) }\end{array}$ & 6 & 2 & 4 & 25 & 2 \\
\hline & $\begin{array}{c}\text { TFPO-CSP } \\
(\mathrm{ABC})\end{array}$ & 9 & 2 & 8 & 8 & 2 \\
\hline & $\begin{array}{c}\text { TFPO-CSP } \\
\text { (GA) }\end{array}$ & 10 & 1 & 2 & 3 & 2 \\
\hline \multirow{3}{*}{ Maximum } & $\begin{array}{l}\text { TFPO-CSP } \\
\text { (PSO) }\end{array}$ & 35 & 17 & 15 & 35 & 17 \\
\hline & $\begin{array}{c}\text { TFPO-CSP } \\
(\mathrm{ABC})\end{array}$ & 14 & 9 & 14 & 35 & 6 \\
\hline & $\begin{array}{c}\text { TFPO-CSP } \\
\text { (GA) }\end{array}$ & 35 & 1 & 8 & 15 & 12 \\
\hline
\end{tabular}

Table 4 The average, minimum and maximum number of iterations taken by TFPO-CSP (PSO), TFPO-CSP (GA) and TFPO-CSP (ABC) approach to select the temporal filter parameters (Dataset 2)

\begin{tabular}{|c|c|c|c|c|c|c|c|c|}
\hline \multirow{2}{*}{ Iterations } & \multirow{2}{*}{ Method } & \multicolumn{5}{|c|}{ Subject } & \multirow[b]{2}{*}{$f$} & \multirow[b]{2}{*}{$g$} \\
\hline & & $a$ & $b$ & $c$ & $d$ & $e$ & & \\
\hline \multirow{3}{*}{ Minimum } & $\begin{array}{c}\text { TFPO-CSP } \\
\text { (PSO) }\end{array}$ & 1 & 1 & 1 & 5 & 1 & 1 & 1 \\
\hline & $\begin{array}{c}\text { TFPO-CSP } \\
\text { (ABC) }\end{array}$ & 1 & 1 & 1 & 1 & 1 & 1 & 1 \\
\hline & $\begin{array}{l}\text { TFPO-CSP } \\
\text { (GA) }\end{array}$ & 1 & 1 & 1 & 1 & 1 & 1 & 1 \\
\hline \multirow{3}{*}{ Average } & $\begin{array}{l}\text { TFPO-CSP } \\
\text { (PSO) }\end{array}$ & 1 & 2 & 5 & 14 & 5 & 4 & 1 \\
\hline & $\begin{array}{l}\text { TFPO-CSP } \\
(\mathrm{ABC})\end{array}$ & 5 & 1 & 2 & 1 & 5 & 5 & 1 \\
\hline & $\begin{array}{c}\text { TFPO-CSP } \\
\text { (GA) }\end{array}$ & 3 & 1 & 1 & 1 & 2 & 2 & 1 \\
\hline \multirow{3}{*}{ Maximum } & $\begin{array}{l}\text { TFPO-CSP } \\
\text { (PSO) }\end{array}$ & 4 & 15 & 21 & 22 & 12 & 8 & 7 \\
\hline & $\begin{array}{c}\text { TFPO-CSP } \\
\text { (ABC) }\end{array}$ & 35 & 4 & 8 & 5 & 17 & 21 & 8 \\
\hline & $\begin{array}{c}\text { TFPO-CSP } \\
(\mathrm{GA})\end{array}$ & 25 & 1 & 10 & 2 & 18 & 15 & 1 \\
\hline
\end{tabular}

Fig. 5 shows the surface plot in 3D space of the classification accuracies (for subject $a a$ of dataset 1) when lower and upper cutoff frequencies are varied with a fixed filter order set at 8. It shows that the frequency band selected has a direct impact on the overall performance of the system and justifies the need for searching for filter band parameters. A trace of how a chromosome (for subject $a a$ of dataset 1) moves through the search space (for TFPO-CSP (GA)) in order to get to the optimal solution for one of the trial runs is also shown in Fig. 5 with black and red circles indicating the start and end points, respectively. It can be seen from Fig. 5 that the TFPO-CSP (GA) algorithm maneuvers through the search space and successfully finds the optimal solution (shown as connecting lines).

Fig. 5 Surface plot showing accuracies for different frequency band combinations with filter order fixed at 8 using dataset 1 (subject $a a$ ). An example of the trace of how a chromosome moves through the search space for TFPO-CSP (GA) is also shown with black and red circles indicating start and end points

To further verify the performance of our predictor with GA as the optimization algorithm (TFPO-CSP (GA)), the power spectrum density (PSD) of the 2 class MI EEG data is conducted. The average PSD of each task for all five subjects derived over trials using all channels data is shown in Fig. 6. It can be noted that the proposed TFPO-CSP (GA) approach effectively searched for a filter band that gives minimum error rate between the two tasks i.e. the frequency band in which the difference in PSD of the 2 tasks is largest. This is further explained in Fig. 5 where it is shown that the particle successfully selects the filter parameters that give the optimal solution. Thus the findings reveal that the most responsive frequency band varies from subject to subject. This explains the need for automatic subject-specific frequency band selection for optimal performance.

Fig. 6 Average power spectrum density of the EEG dataset for the five subjects of dataset 1 (using all the channels data)

\section{Discussion}

To further show the effectiveness of the proposed method, Fig. 7 shows the topographic plot of the spatial filters that are learned using the CSP and TFPO-CSP methods (spatial filters learned during one of the random runs using subject $a w$ of dataset 1). It can be seen that the spatial filters learned by the TFPO-CSP method highly correlates with the two MI tasks (right hand and left foot) as they are contralaterally well localized in the left and right sensorimotor cortex regions. We also tested the classification performance of the learned spatial filters of subject aw shown in Fig. 7 and obtained $89.29 \%$ and $96.43 \%$ classification accuracy (on test data) using CSP and TFPO-CSP spatial filters, respectively. This further validates the results that the spatial filters learned by TFPO-CSP are able to more effectively discriminate between the two MI tasks compared to the spatial filters learned by CSP. This improved performance is due to the use of the most responsive subject-dependent frequency band, which is 
automatically determined using our proposed TFPO-CSP predictor.

Fig. 7 The learned spatial filters for CSP and TFPO-CSP methods for subject $a w$ of dataset 1 (for one of the random trial runs)

In order to keep the computation complexity of the proposed method to a minimum, we have employed a single frequency band. Thus the time (test time) taken for a single trial to be processed (temporal filtering, spatial filtering, and feature extraction) and classified using the trained classifier for different methods is shown in Table 5. The time calculated are the test time and does not include the training time as mostly offline training is performed for BCI applications. It should be noted that as the number of bands used is increased, the computation time also increases i.e. computation time is directly proportional to the number of bands used for processing. Our proposed approach uses a single frequency band and thus has a lower computation time compared to that of CSSP, FBCSP, DFBCSP, and SBLFB. The computation time of TFPO-CSP (GA) is higher than that of CSP due to the fact that filter order of 30 (maximum) is used for computing the time for TFPOCSP (GA) approach. If the filter order is same as that of CSP then the time for CSP and TFPO-CSP (PSO) methods would also be same. The test time for TFPOCSP (ABC) and TFPO-CSP (PSO) will be same as that of TFPO-CSP (PSO) if same filter orders are used. Thus our approach can be top choice for use with wearable devices, which require algorithms that are computationally less expensive so that it can last longer as they are battery powered. Matlab running on a personal computer at $3.3 \mathrm{GHz}$ (Intel(R) Core(TM) i3) has been used for all processing.

Table 5 Test time required by different algorithms for single trial MI EEG signal classification

\begin{tabular}{lcccccc}
\hline Method & CSP & CSSP & FBCSP & DFBCSP & SBLFB & $\begin{array}{c}\text { TFPO-CSP } \\
(\mathrm{GA})\end{array}$ \\
\hline Time $(\mathrm{ms})$ & 3.36 & 12.30 & 14.22 & 10.80 & 13.10 & 6.96 \\
\hline
\end{tabular}

The TFPO-CSP predictor with GA is able to successfully find the optimal filter band parameters that give higher accuracy without the use of fitness condition i.e. allowing the algorithm to run for maximum number of iterations. However, the drawback is longer training time. Since the training procedure can be performed offline, this drawback can be overcome. Nonetheless, to reduce the training time, fitness condition has been used to converge to the solution in a faster manner. The setting of different fitness condition for each subject proves vital as the training time is reduced and the proposed TFPO-CSP with GA is still able to achieve higher accuracy. The training time required by TFPOCSP with PSO, TFPO-CSP with GA and TFPO-CSP with $\mathrm{ABC}$ is given in Table 6, where Init is the average initialization time and 1 iter is the average time required by the respective methods for execution of one complete iteration. Therefore, the overall average training time for each of the methods will be the initialization time plus the time taken for one iteration multiplied by the average number of iterations required by the respective methods. On overall, the TFPO-CSP (GA) approach requires the minimum number of iterations to search for the optimal parameters. Therefore, the TFPO-CSP (GA) approach is recommended over TFPO-CSP (ABC) and TFPO-CSP (PSO).

Table 6 Initialization time and time taken for one iteration during training that is required by different algorithms

\begin{tabular}{ccccccc}
\hline Method & \multicolumn{2}{c}{ TFPO-CSP (PSO) } & \multicolumn{2}{c}{ TFPO-CSP (GA) } & \multicolumn{2}{c}{ TFPO-CSP (ABC) } \\
\hline & Init & 1 iter & Init & 1 iter & Init & 1 iter \\
Time (s) & 145.44 & 159.18 & 128.90 & 128.95 & 72.97 & 145.44 \\
\hline
\end{tabular}

Our proposed predictor obtained the overall minimum error rates of $10.19 \%$ and $19.92 \%$ for dataset 1 and dataset 2, respectively. It outperformed all competing methods evaluated in this work achieving an improvement of $3.54 \%$ and $0.65 \%$ compared to SBLFB method for dataset 1 and dataset 2 , respectively. In comparison with SFBCSP and DFBCSP our predictor achieved an improvement of $4.22 \%$ and $0.75 \%$ for dataset 1 while also achieving an improvement of 7.29\% and $2.94 \%$ for dataset 2, respectively. Our proposed predictor also outperformed the sparsity-aware method [47] (where weighted averaging has been introduced for estimating the average covariance matrix, which is used for calculating the CSP spatial filters) that was evaluated on dataset 1, achieving an improvement of $2.44 \%$. However, the sparsity-aware method performed well compared to CSP method, and can be incorporated in our predictor, which might further improve the performance of the system.

It can be noted that for some of the subjects the lower cutoff frequencies were in the range of $2-8 \mathrm{~Hz}$, which is lower than the mu rhythm. The proposed method only finds the band that gives optimal performance and guarantees that the selected frequency band contains most of the useful information. However, it does not guarantee that all the frequencies present in the selected 
frequency band contain useful information. While searching for the optimal parameters, it is possible for the proposed method to find a suitable solution that has relatively lower value for lower cutoff frequency parameter depending on the search space through which the algorithm maneuvers to find the optimal parameters. It is possible that no important information is contained by the signal in the lower frequencies and having or not having these will make no notable difference in the performance. However, it should also be noted that very low values of lower cutoff frequency parameter has been obtained using all the three optimization algorithms for subjects $a l$ and $a y$ of dataset 1 and subject $g$ of dataset 2 . This suggests that there is some information in the lower frequencies, which is causing all the optimization algorithms used to select the lower value for lower cutoff frequency. Since both datasets have been recorded from healthy subjects, there is very little chance of abnormal behavior. Thus, further research needs to be carried out in order to investigate if these lower frequencies actually contain any useful information about the different MI tasks. However, according to [11] signal in the delta band can be caused by some continuous-attention tasks and signal in theta band can be caused due to a person actively trying to repress an action. Since the trials in both the datasets are cue based and the subjects are required to imagine the MI tasks, it is highly likely that these can be the reason leading to the selection of low values for lower cutoff frequency parameter for some of the subjects.

Furthermore, we have performed paired t-test with 5\% significance level to show the significance of the classification error rate of the proposed method compared to the top 2 performing methods (SBLFB and DFBCSP). The p-value obtained was 0.045 and 0.034 (compared to SBLFB and DFBCSP, respectively), which shows that significant improvements have been achieved.

The main advantage of the proposed TFPO-CSP predictor is that it automatically finds the responsive frequency band, which contains significant information for maximum discrimination between the MI tasks. This also results in the spatial filter learned to be more effective in discriminating between the different MI tasks. Furthermore, the TFPO-CSP predictor is able to achieve enhanced performance using only a single frequency band compared to the competing methods that utilize multiple sub-bands. This accounts for the low computational complexity of the method and makes it more suitable for implementation on wearable devices. This can help in the development of low cost BCI systems that will assist people with disabilities to recover their environmental control capabilities. It can also be beneficial in epileptic seizure detection using EEG signals as being able to predict/detect pre-seizure more accurately will save more lives and prevent more accidents. The disadvantage of the TFPO-CSP predictor is that it requires a longer training time compared to other competing methods. However, this problem can be overcome by training the model offline. Moreover, being able to effectively find filter parameters that will produce optimal results in a quicker way is desirable for BCI applications that will require online training. To add on, the range of filter order proposed in this work is specifically for butterworth filter. If other types of filters are to be used, then the users will need to investigate and determine the range for the filter order as different types of filters behave differently. The users can also simply select maximum range up to 50 and the proposed TFPOCSP predictor will automatically find the best value. However, it might take longer time to find the optimal parameters given a larger search space.

Moreover, the proposed method can be utilized for further tuning the sub-bands selected by other approaches (such as SBCSP, FBCSP, DFBCSP, SFBCSP, SBLFB and SFTOFSRC) and using dimensionality reduction or feature selection techniques [32-41] to remove redundant features, which can further improve the performance of the existing methods. On the other hand, the use of dictionary optimization together with SRC can further enhance the performance of the TFPO-CSP predictor.

\section{Conclusion}

In this paper, we proposed a new automatic method of finding the temporal filter parameters for improved MI EEG signal classification. The parameters of a butterworth filter are tuned for optimal performance. The proposed method performed well in selecting subjectspecific frequency bands and outperformed state-of-theart methods achieving the lowest classification error rates. The only drawback of the approach is that it requires a longer training time compared to other methods. However, this drawback can be avoided since training is usually performed offline for BCI applications. The proposed methods capability in finding the temporal filter parameters that gives optimal performance in terms of MI EEG signal classification together with its low computational complexity (for classification of test samples) makes it a strong candidate for future BCI systems.

Acknowledgements Special thanks to the editors and anonymous reviewers for their positive and constructive 
comments and suggestions that helped improve our manuscript. This work was supported by the Faculty Research Committee of the University of the South Pacific, Laboratory for Medical Science Mathematics, RIKEN Center for Integrative Medical Sciences, Yokohama 230-0045, Japan and College Research Committee of Fiji National University.

Algorithm For the benefit of the research community, the TFPO-CSP algorithm (MATLAB m-file) is available on our website: http://www.alok-ai-lab.com/publications.php.

\section{References}

1. Akram F, Metwally MK, Hee-Sok H, Hyun-Jae J, TaeSeong K (2013) A novel P300-based BCI system for words typing. In: 2013 International Winter Workshop on BrainComputer Interface (BCI). pp 24-25. doi: 10.1109/IWWBCI.2013.6506617

2. Andrew C, Pfurtscheller G (1997) On the existence of different alpha band rhythms in the hand area of man. Neurosci Lett 222:103-106. doi: https://doi.org/10.1016/S0304-3940(97)13358-4

3. Ang KK, Chin ZY, Zhang H, Guan C (2008) Filter Bank Common Spatial Pattern (FBCSP) in Brain-Computer Interface. In: IEEE International Joint Conference on Neural Networks. pp 2390 - 2397. doi: 10.1109/IJCNN.2008.4634130

4. Blankertz B, Dornhege G, Krauledat M, Müller KR, Curio $G$ (2007) The non-invasive Berlin Brain-Computer Interface: Fast acquisition of effective performance in untrained subjects. NeuroImage 37:539-550. doi: 10.1016/j.neuroimage.2007.01.051

5. Cincotti F, Mattia D, Aloise F et al (2008) Non-invasive brain-computer interface system: Towards its application as assistive technology. Brain Res Bull 75:796-803. doi: 10.1016/j.brainresbull.2008.01.007

6. Dornhege G, Blankertz B, Curio G, Muller K (2004) Boosting bit rates in noninvasive EEG single-trial classifications by feature combination and multiclass paradigms. IEEE Trans Biomed Eng 51:993-1002. doi: 10.1109/TBME.2004.827088

7. Dornhege G, Blankertz B, Krauledat M, Losch F, Curio G, Muller KR (2006) Combined Optimization of Spatial and Temporal Filters for Improving Brain-Computer Interfacing. IEEE Trans Biomed Eng 53:2274-2281. doi: 10.1109/TBME.2006.883649

8. Higashi H, Tanaka T (2013) Simultaneous Design of FIR Filter Banks and Spatial Patterns for EEG Signal Classification. IEEE Trans Biomed Eng 60:1100-1110. doi: 10.1109/TBME.2012.2215960

9. Janjarasjitt, S. (2017). Epileptic seizure classifications of single-channel scalp EEG data using wavelet-based features and SVM. Med Biol Eng Comput 55:1743-1761. doi: 10.1007/s11517-017-1613-2

10. Karaboga D (2005) An idea based on honey bee swarm for numerical optimization. Technical Report TR06: Erciyes University.

11. Kirmizi-Alsan E, Bayraktaroglu Z, Gurvit H, Keskin YH, Emre M, Demiralp T (2006) Comparative analysis of event-related potentials during $\mathrm{Go} / \mathrm{NoGo}$ and $\mathrm{CPT}$ : Decomposition of electrophysiological markers of response inhibition and sustained attention. Brain Res 1104:114-128. doi: 10.1016/j.brainres.2006.03.010

12. Kleih SC, Kuafmann T, Zickler C et al (2011) Out of the frying pan into the fire-the P300-based BCI faces real- 
world challenges. Prog Brain Res 194:27-46. doi: 10.1016/B978-0-444-53815-4.00019-4

13. Koles ZJ (1991) The quantitative extraction and topographic mapping of the abnormal components in the clinical EEG. Electroencephalography Clin Neurophysiol 79:440-447. doi: https://doi.org/10.1016/00134694(91)90163-X

14. Kumar S, Mamun K, Sharma A (2017) CSP-TSM: Optimizing the performance of Riemannian tangent space mapping using common spatial pattern for MI-BCI. Comput Biol Med 91:231-242. doi: 10.1016/j.compbiomed.2017.10.025

15. Kumar S, Sharma A, Mamun K, Tsunoda T (2016) A Deep Learning Approach for Motor Imagery EEG Signal Classification. In: 3rd Asia-Pacific World Congress on Computer Science and Engineering. doi: 10.1109/APWCon-CSE.2016.017

16. Kumar S, Sharma A, Tsunoda T (2017) An improved discriminative filter bank selection approach for motor imagery EEG signal classification using mutual information. BMC Bioinformatics 18:545. doi: 10.1186/s12859-017-1964-6

17. Kumar S, Sharma R, Sharma A, Tsunoda T (2016) Decimation Filter with Common Spatial Pattern and Fishers Discriminant Analysis for Motor Imagery Classification. In: IEEE World Congress on Computational Intelligence. doi: 10.1109/IJCNN.2016.7727457

18. La Rocca D, Campisi P, Sole-Casals J (2013) EEG based user recognition using BUMP modelling. In: International Conference of the Biometrics Special Interest Group (BIOSIG). pp 1-12.

19. Lemm S, Blankertz B, Curio G, Muller K (2005) Spatiospectral filters for improving the classification of single trial EEG. IEEE Trans Biomed Eng 52:1541-1548. doi: 10.1109/TBME.2005.851521

20. McFarland DJ, Sarnacki WA, Wolpaw JR (2010) Electroencephalographic (EEG) control of threedimensional movement. J Neural Eng 7:036007. doi: 10.1088/1741-2560/7/3/036007

21. Miao M, Wang A, Liu F (2017) A spatial-frequencytemporal optimized feature sparse representation-based classification method for motor imagery EEG pattern recognition. Med Biol Eng Comput 55:1589-1603. doi: 10.1007/s11517-017-1622-1

22. Mumtaz W, Ali SSA, Yasin MAM, Malik AS (2018) A machine learning framework involving EEG-based functional connectivity to diagnose major depressive disorder (MDD). Medi Biol Eng Comput. 56:233-246. doi: 10.1007/s11517-017-1685-z

23. Naveen, R.S., and Julian, A. (2013). Brain computing interface for wheel chair control. In: Fourth International
Conference on Computing, Communications and Networking Technologies (ICCCNT). pp 1-5. doi: 10.1109/ICCCNT.2013.6726572

24. Nicolas-Alonso LF, Gomez-Gil J (2012) Brain Computer Interfaces, a Review. Sensors 12:1211. doi: $10.3390 / \mathrm{s} 120201211$

25. Novi Q, Cuntai G, Dat TH, Ping X (2007) Sub-band Common Spatial Pattern (SBCSP) for Brain-Computer Interface. In: 3rd International IEEE/EMBS Conference on Neural Engineering. pp 204-207. doi: 10.1109/CNE.2007.369647

26. Pfurtscheller G, Lopes da Silva FH (1999) Event-related EEG/MEG synchronization and desynchronization: basic principles. Clin Neurophysiol 110:1842-1857. doi: https://doi.org/10.1016/S1388-2457(99)00141-8

27. Poli R, Kennedy J, Blackwell T (2007) Particle swarm optimization. Swarm Intelligence 1:33-57.

28. Ramesh S, Krishna MG, Nakirekanti M (2014) Brain Computer Interface System for Mind Controlled Robot using Bluetooth. Int J Comput Appl 104:20-23.

29. Ramoser H, Muller-Gerking J, Pfurtscheller G (2000) Optimal spatial filtering of single trial EEG during imagined hand movement. IEEE Trans Rehabil Eng 8. doi: 10.1109/86.895946

30. Samiee K, Kovcs P, Gabbouj M (2017) Epileptic seizure detection in long-term EEG records using sparse rational decomposition and local Gabor binary patterns feature extraction. Knowledge Based Syst 118:228-240. doi: 10.1016/j.knosys.2016.11.023

31. Serruya MD (2014) Bottlenecks to clinical translation of direct brain-computer interfaces. Front Syst Neurosci 8:226. doi: 10.3389 /fnsys. 2014.00226

32. Sharma A, Imoto S, Miyano S (2012) A between-class overlapping filter-based method for transcriptome data analysis. J Bioinform Comput Biol 10:1250010. doi: $10.1142 / \mathrm{S} 0219720012500102$

33. Sharma A, Imoto S, Miyano S (2012) A filter based feature selection algorithm using null space of covariance matrix for DNA microarray gene expression data. Current Bioinform 7:289-294. doi: 10.2174/157489312802460802

34. Sharma A, Imoto S, Miyano S (2012) A Top-r Feature Selection Algorithm for Microarray Gene Expression Data. IEEE/ACM Trans Comput Biol Bioinform 9:754-764. doi: 10.1109/TCBB.2011.151

35. Sharma A, Imoto S, Miyano S, Sharma V (2012) Null space based feature selection method for gene expression data. Int $\mathrm{J}$ Mach Learn Cybernetics 3:269-276. doi: https://doi.org/10.1007/s13042-011-0061-9

36. Sharma A, Koh C, Imoto S, Miyano S (2011) Strategy of finding optimal number of features on gene expression data. Electron Lett 47:480-482. doi: 10.1049/el.2011.0526 
37. Sharma A, Paliwal KK (2015) A deterministic approach to regularized linear discriminant analysis. Neurocomputing 151:207-214.

doi:

https://doi.org/10.1016/j.neucom.2014.09.051

38. Sharma A, Paliwal KK (2007) Fast principal component analysis using fixed-point algorithm. Pattern Recogn Lett 28:1151-1155. doi: https://doi.org/10.1016/j.patrec.2007.01.012

39. Sharma A, Paliwal KK (2015) Linear discriminant analysis for the small sample size problem: an overview. Int J Mach Learn Cybernetics 6:443-454. doi: https://doi.org/10.1007/s13042-013-0226-9

40. Sharma A, Paliwal KK (2012) A new perspective to null linear discriminant analysis method and its fast implementation using random matrix multiplication with scatter matrices. Pattern Recogn 45:2205-2213. doi: https://doi.org/10.1016/j.patcog.2011.11.018

41. Sharma A, Paliwal KK, Imoto S, Miyano S (2013) Principal component analysis using QR decomposition. Int $\mathrm{J}$ Mach Learn Cybernetics 4:679-683. doi: https://doi.org/10.1007/s13042-012-0131-7

42. Shin Y, Lee S, Ahn M, Cho H, Jun SC, Lee H-N (2015) Simple adaptive sparse representation based classification schemes for EEG based brain-computer interface applications. Comput Biol Med 66:29-38. doi: 10.1016/j.compbiomed.2015.08.017

43. Silvoni S, Ramos-Murguialday A, Cavinato M et al (2011) Brain-Computer Interface in Stroke: A Review of Progress. Clin EEG Neurosci 42:245-252. doi: 10.1177/155005941104200410

44. Sohrabpour A, Lu Y, Kankirawatana P, Blount J, Kim H, He B (2015) Effect of EEG electrode number on epileptic source localization in pediatric patients. Clin Neurophysiol 126:472-480. doi: 10.1016/j.clinph.2014.05.038

45. Suk HI, Lee SW (2013) A Novel Bayesian Framework for Discriminative Feature Extraction in Brain-Computer Interfaces. IEEE Trans Pattern Anal Mach Intell 35:286299. doi: 10.1109/TPAMI.2012.69

46. Thomas KP, Cuntai G, Lau CT, Vinod AP, Keng AK. (2009). A New Discriminative Common Spatial Pattern Method for Motor Imagery Brain Computer Interfaces. IEEE Trans Biomed Eng 56:2730-2733. doi: 10.1109/TBME.2009.2026181

47. Tomida N, Tanaka T, Ono S, Yamagishi M, Higashi H (2015) Active Data Selection for Motor Imagery EEG Classification. IEEE Trans Biomed Eng 62:458-467. doi: 10.1109/TBME.2014.2358536

48. Wang H, Zhang Y, Waytowich NR et al (2016) Discriminative Feature Extraction via Multivariate Linear Regression for SSVEP-Based BCI. IEEE Trans Neural Syst Rehabil Eng 24:532-541. doi: 10.1109/TNSRE.2016.2519350
49. Wei Q, Wei Z (2015) Binary particle swarm optimization for frequency band selection in motor imagery based braincomputer interfaces. Bio-Med Mater Eng 26:S1523-S1532. doi: 10.3233/BME-151451

50. Widmann A, Schröger E (2012) Filter Effects and Filter Artifacts in the Analysis of Electrophysiological Data. Front Psychol 3. doi: 10.3389/fpsyg.2012.00233

51. Woehrle H, Krell MM, Straube S, Kim SK, Kirchner EA, Kirchner F (2015) An Adaptive Spatial Filter for User-Independent Single Trial Detection of Event-Related Potentials. IEEE Trans Biomed Eng 62:1696-1705. 10.1109/TBME.2015.2402252

52.Xu Y, Fan P, Yuan L (2013) A Simple and Efficient Artificial Bee Colony Algorithm. Mathematical Probl Eng 9. doi:

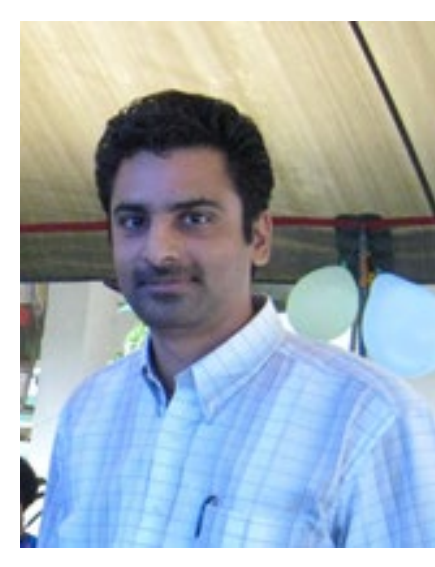
http://dx.doi.org/10.1155/2013/526315

53. Yu T, Xiao J, Wang $F$ et al (2015) Enhanced Motor Imagery Training Using a Hybrid BCI With Feedback. IEEE Trans Biomed Eng 62:1706-1717. doi: 10.1109/TBME.2015.2402283

54.Zhang Y, Wang Y, Jin J, Wang X (2017) Sparse Bayesian Learning for Obtaining Sparsity of EEG Frequency Bands Based Feature Vectors in Motor Imagery Classification. Int J Neural Syst 27:1650032. doi: 10.1142/S0129065716500325

55.Zhang Y, Wang Y, Zhou G, Jin J, Wang B, Wang X, Cichocki A (2018) Multi-kernel extreme learning machine for EEG classification in brain-computer interfaces. Expert Systems with Applications 96:302-310. doi: https://doi.org/10.1016/j.eswa.2017.12.015

56.Zhang Y, Zhou G, Jin J, Wang X, Cichocki A (2015) Optimizing spatial patterns with sparse filter bands for motor-imagery based brain-computer interface. J Neurosci Methods 255:85-91. doi: 10.1016/j.jneumeth.2015.08.004

57.Zhang Y, Zhou G, Jin J, Zhao Q, Wang X, Cichocki A (2016) Sparse Bayesian Classification of EEG for Brain Computer Interface. IEEE Trans Neural Netw Learn syst 27:2256-2267. doi: 10.1109/TNNLS.2015.2476656

58.Zhou G, Zhao Q, Zhang Y, Adalı T, Xie S, Cichocki A (2016) Linked Component Analysis From Matrices to High-Order Tensors: Applications to Biomedical Data. Proceedings of the IEEE 104:310-331. doi: 10.1109/JPROC.2015.2474704 


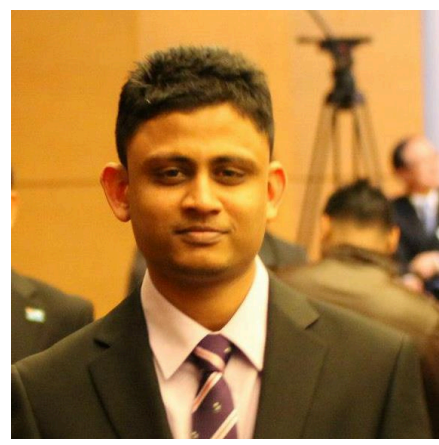

Shiu Kumar is currently pursuing his $\mathrm{Ph} . \mathrm{D}$. degree at School of Engineering \& Physics, The University of the South Pacific, Fiji. His research interests include brain-computer interface, bio-medical signal processing, and machine learning.

Alok Sharma received the Ph.D. degree in the area of pattern recognition from Griffith University, Brisbane, Australia in 2006. He is a Scientist at RIKEN Center for Integrative Medical Sciences, Japan. He is also an Adjunct Associate Professor at the Institute for Integrated and Intelligent Systems (IIIS), Griffith University, Australia. He is concurrently serving as a Professor at USP. His research interests include pattern recognition, human cancer classification, and proteomics. Website: www.alok-ai-lab.com 